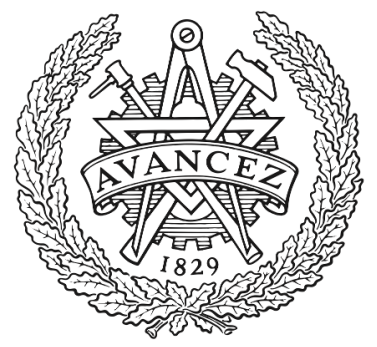

CHALMERS

UNIVERSITY OF TECHNOLOGY

\title{
A time domain model for the wheel/rail interaction aiming to include non-linear contact stiffness and tangential friction
}

Downloaded from: https://research.chalmers.se, 2023-04-26 12:02 UTC

Citation for the original published paper (version of record):

Pieringer, A., Kropp, W., Nielsen, J. (2007). A time domain model for the wheel/rail interaction aiming to include non-linear contact stiffness and tangential friction. Proceedings of the 9th International Workshop on Railway Noise (IWRN9),Munich, Germany, 2007 (published on CD)

N.B. When citing this work, cite the original published paper. 


\title{
A time domain model for wheel/rail interaction aiming to include non-linear contact stiffness and tangential friction
}

\author{
A. Pieringer ${ }^{1, *}$, W. Kropp ${ }^{1}$, J.C.O. Nielsen ${ }^{2}$ \\ ${ }^{1}$ Division of Applied Acoustics / CHARMEC, Chalmers University of Technology, \\ SE-412 96 Göteborg, Sweden \\ ${ }^{2}$ Department of Applied Mechanics / CHARMEC, Chalmers University of Technology, \\ SE-412 96 Göteborg, Sweden \\ *Tel: +46 31772 2209, Fax: +46 31772 2212, E-mail: astrid.pieringer@chalmers.se
}

\begin{abstract}
A time domain model for the dynamic interaction between railway wheel and rail, which takes into account the non-linear processes in the contact zone and aims at predicting both normal and tangential contact forces, is presented. The model follows an approach that successfully has been used for instance for the modelling of the interaction between road and tyre.

Track and wheel are described as linear systems by the means of impulse response functions. The contact zone is modelled by non-linear contact springs depending on the roughness of rail and wheel. Here, the method of the area of real contact is applied in order to obtain the required spring characteristics. For the tangential contact, a characteristic function for the friction coefficient is applied.

In a first stage, the approach is demonstrated for the calculation of contact forces normal to the surfaces of rail and wheel. For validation, the results from the model are compared with an existing time domain model that itself has been validated by field testing. Very good agreement is found for different types of roughness excitation. The model is then used to evaluate the contact filter effect for a rail with sinusoidal corrugation. No significant difference is observed between quasi-static and dynamic roughness filtering.
\end{abstract}

\section{Introduction}

The interaction between wheel and rail is considered as the dominating source for noise emission from railway operations over a wide speed range. The importance of motive power noise is confined to low speeds and aerodynamic noise becomes significant only for high-speed trains. The modelling of the dynamic wheel/rail interaction is therefore a crucial module in railway noise prediction models.

On one hand, this interaction concerns contact forces in the normal direction to the surfaces, caused by the roughness on rail and wheel, giving rise to rolling noise. On the other hand, it regards tangential forces caused by the friction between rail and wheel seen as responsible for squealing noise.

During the past decades, models of different comprehensiveness have been published. The most wide spread prediction model for railway noise is a frequency domain model developed by Remington [1] and Thompson [2]. It is in the nature of frequency domain models that they can only include a linear contact model. In order to study non-linearities in the normal wheel/rail interaction, Wu and Thompson applied a moving irregularity model in the time domain. They found that non-linearities cannot be neglected in the cases of a severely corrugated rail and/or a low static contact preload [3]. In general, this statement holds in cases where loss of contact is likely to occur including for instance operating with wheel flats [4] and passing over rail joints [5]. These results have been confirmed 
by Nordborg who used both a frequency domain model and a time domain model based on Green's functions to study non-linear effects in the normal interaction [6]. He also found that the inclusion of parametric excitation due to discrete supports is important for lower frequencies around the sleeperpassing frequency and for higher frequencies around the pinned-pinned resonance frequency of the rail.

While a considerable number of models dealing with the normal wheel/rail interaction have been published, only a few models predicting the coupled normal and tangential interaction are available. $\mathrm{Wu}$ and Thompson extended their time domain model to include the lateral wheel/rail interaction [7]. The coupling between normal and lateral directions was introduced through the track dynamics due to an offset of the wheel/rail contact point from the rail centre line which is assumed as an input to the model.

In contrast to frequency domain models, time domain models can include non-linearities. However, in general, they require much more computational effort. To surmount this shortcoming, a computationally efficient time domain model that aims to include the coupled normal and longitudinal/lateral wheel/rail interaction is presented in this paper. Wheel and rail are represented by impulse response functions (Green's functions). The contact model is non-linear and allows for loss of contact. In order to obtain the required contact spring characteristics, the area of real contact of the rough surfaces is evaluated. The contact filtering effect, which consists in the attenuation of high frequency excitation due to the finite size of the wheel/rail contact, is hereby integrated into the model in a natural manner. The generality of the approach separating wheel, rail and contact in different modules, allows to embed any wheel or rail model that can be represented by Green's functions. Choosing a rail model including discrete supports therefore enables the consideration of parametric excitation without difficulties. A major advantage of the approach is furthermore that the Green's functions are precalculated and by this means calculation times for simulations are kept low.

The described concept has already been successfully applied in the area of tyre/road noise, see e.g. [8]. The tyre/road contact differs in several ways from the wheel/rail contact. One of the main differences is the relatively short wavelengths for waves propagating on the tyre structure which demand a fine discretisation of the contact patch. This leads to a substantial increase in computational effort in comparison to the simulation of the wheel/rail interaction where the wavelengths are long in comparison to the contact patch and only a few contact points are needed. Despite these differences, one can expect that the approach will work in a similar way.

In the area of wheel/rail contact the utilisation of Green's functions goes back to Heckl's proposal for a railway simulation program [9]. Subsequently, this approach has e.g. been used by Nordborg [6].

In section 2, the interaction model for normal contact is described and the applied track and wheel models are presented. Furthermore, it is shown how the non-linear contact stiffness is calculated with the method of the area of real contact and how the model can be extended to include tangential contact. For validation, simulation results of the normal interaction model are compared to results from the train/track interaction model DIFF [10] in section 3. This model, developed by Nielsen and Igeland, has been validated by field testing [11]. In section 4, the presented model is applied to evaluate the contact filter effect for passing over a rail with sinusoidal corrugation.

\section{Wheel/rail interaction model}

The wheel/rail interaction model is a time domain model based on the moving vehicle model approach. Rail and wheel are modelled by Green's functions. 


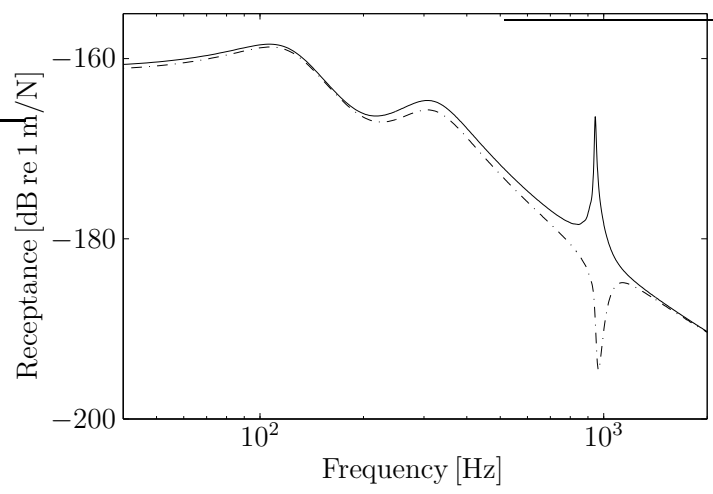

(a) Magnitude of the point receptance.

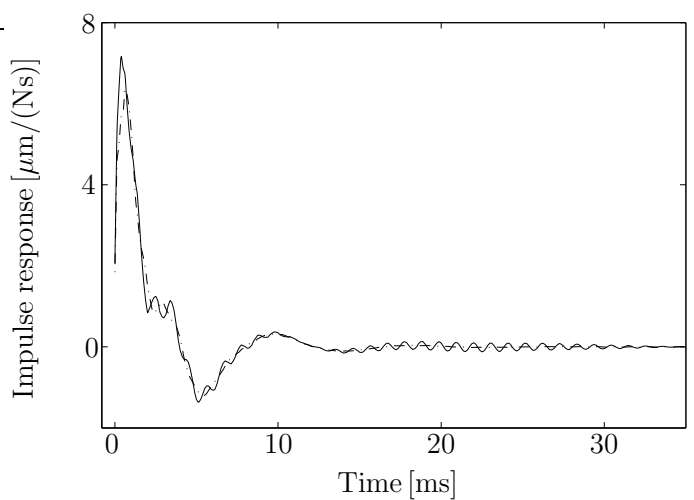

(b) Moving Green's function for $v=100 \mathrm{~km} / \mathrm{h}$.

Fig. 1. Response of the track. - excitation at midspan between two sleeper positions, $-\cdot-\cdot$ excitation above a sleeper position.

\subsection{Track and wheel model}

The track model used in this study is a linear finite element model accounting for discrete supports consisting of railpads and sleepers on ballast. Rail and sleepers are modelled by RayleighTimoshenko beam elements. The track model contains 70 sleeper bays and it has clamped ends at both boundaries. Fig. 1(a) shows the magnitude of the track point receptance for two different excitation positions. For excitation at midspan between two sleeper positions, a sharp peak is observed at $945 \mathrm{~Hz}$, which is the pinned-pinned resonance frequency. The point receptance for excitation over a sleeper shows correspondingly an anti-resonance in this frequency range, slightly shifted to higher frequencies. A detailed description of the track model is given in references [10] and [12].

For inclusion into the wheel/rail interaction model, the track has to be represented by so-called moving Green's functions $\tilde{g}_{\mathrm{R}, v}^{x_{0}}(t)$ [6]. The function $\tilde{g}_{\mathrm{R}, v}^{x_{0}}(t)$ describes, for excitation of the rail (index $\mathrm{R})$ at the position $x_{0}$ at time $t_{0}=0$, the displacement response of the rail at a point moving with train speed $v$ away from the excitation, thus at the contact point between wheel and rail. The discrete version of $\tilde{g}_{\mathrm{R}, v}^{x_{0}}(t)$, denoted $g_{\mathrm{R}, v}^{x_{0}}(n)$, is constructed from a series of track receptances $\tilde{G}_{\mathrm{R}}^{x_{0}, x_{0}+\alpha}(f)$. The superscripts specify the excitation point $x_{0}$ and the response point $x_{0}+\alpha$. The Green's functions $\tilde{g}_{\mathrm{R}}^{x_{0}, x_{0}+\alpha}(t)$ corresponding to the track receptances are obtained by inverse Fourier transform

$$
\tilde{g}_{\mathrm{R}}^{x_{0}, x_{0}+\alpha}(t)=\mathscr{F}^{-1}\left(\tilde{G}_{\mathrm{R}}^{x_{0}, x_{0}+\alpha}(f)\right) .
$$

Exploiting the coupling between time increment $\Delta t$ and space increment $\Delta x, \Delta x=v \Delta t$, the discrete moving Green's functions are constructed as

$$
g_{\mathrm{R}, v}^{x_{0}}(n)=\tilde{g}_{\mathrm{R}}^{x_{0}, x_{0}+n \Delta x}(n \Delta t),
$$

where $n=0,1,2, \ldots, N-1$ and $N$ is the number of samples. Two examples for moving Green's functions are presented in Fig. 1(b). Due to the periodicity of the track, $L / \Delta x$ different moving Green's functions are sufficient to represent the track, where $L$ is the sleeper spacing. With the parameters $L=0.65 \mathrm{~m}$ and $\Delta x=1 \mathrm{~mm}$, this leads to 650 moving Green's functions.

The wheel model used in this study is a simple one. The wheel is represented by a mass $M$ and a primary suspension consisting of a spring with constant $K$ and a viscous damper with constant $C$. The vehicle components above the primary suspension are simplified to a static preload $P$. Treating 


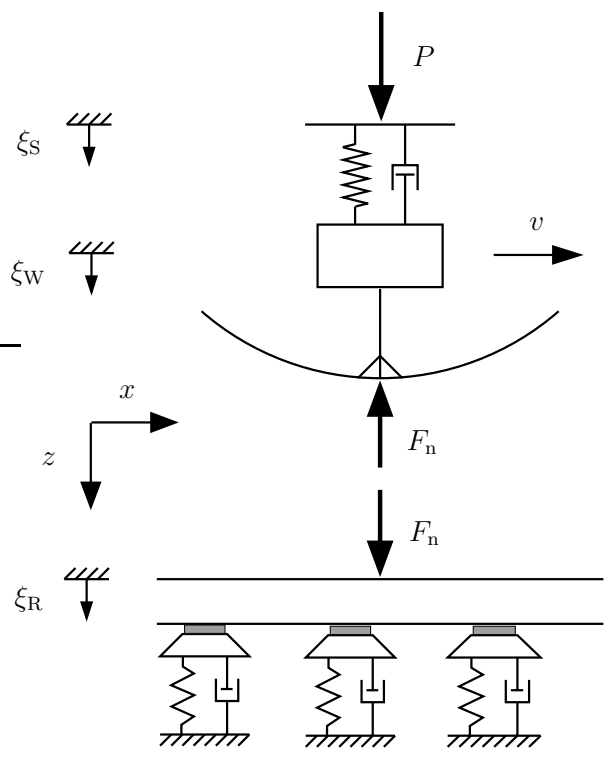

Fig. 2. Wheel/track interaction model.

the wheel as a single degree of freedom system, wheel receptance $\tilde{G}_{\mathrm{W}}(f)$ and wheel Green's function $\tilde{g}_{\mathrm{W}}(t)$ are then obtained as

$$
\begin{aligned}
\tilde{G}_{\mathrm{W}}(f) & =\frac{1}{-(2 \pi f)^{2} M+i(2 \pi f) C+K} \\
\tilde{g}_{\mathrm{W}}(t) & =\mathscr{F}^{-1}\left(\tilde{G}_{\mathrm{W}}(f)\right) .
\end{aligned}
$$

\subsection{Normal contact model}

The wheel/track interaction model is presented schematically in Fig. 2. The wheel moves over the rail in $x$-direction with the constant train speed $v$. The normal contact force, denoted $F_{\mathrm{n}}$, excites the wheel upwards and the rail downwards in the contact point. The static preload $P$ is applied by pressing the wheel onto the rail until the static contact force equals $P$. The suspension is then fixed in the corresponding position $\xi_{\mathrm{S}}$

$$
\xi_{\mathrm{S}}=\xi_{\mathrm{S}}(P) .
$$

The normal displacement of the wheel $\xi_{\mathrm{W}}(t)$ is obtained by convoluting the contact force with the Green's function of the wheel

$$
\xi_{\mathrm{W}}(t)=-\int_{0}^{t} F_{\mathrm{n}}(\tau) \tilde{g}_{\mathrm{W}}(t-\tau) \mathrm{d} \tau+\xi_{\mathrm{S}}=-F_{\mathrm{n}}(t) * \tilde{g}_{\mathrm{W}}(t)+\xi_{\mathrm{S}} .
$$

In a similar manner, the normal displacement of the rail $\xi_{\mathrm{R}}(t)$ is calculated by convoluting the contact force with the moving Green's functions of the rail

$$
\xi_{\mathrm{R}}(t)=\int_{0}^{t} F_{\mathrm{n}}(\tau) \tilde{g}_{\mathrm{R}, v}^{v \tau}(t-\tau) \mathrm{d} \tau \stackrel{\text { def }}{=} F_{\mathrm{n}}(t) * \tilde{g}_{\mathrm{R}, v}^{x_{0}}(t) .
$$

For the calculation of the total normal contact force, the roughness profile in the whole contact patch is considered. For this purpose, a Winkler bedding consisting of independent springs is introduced 


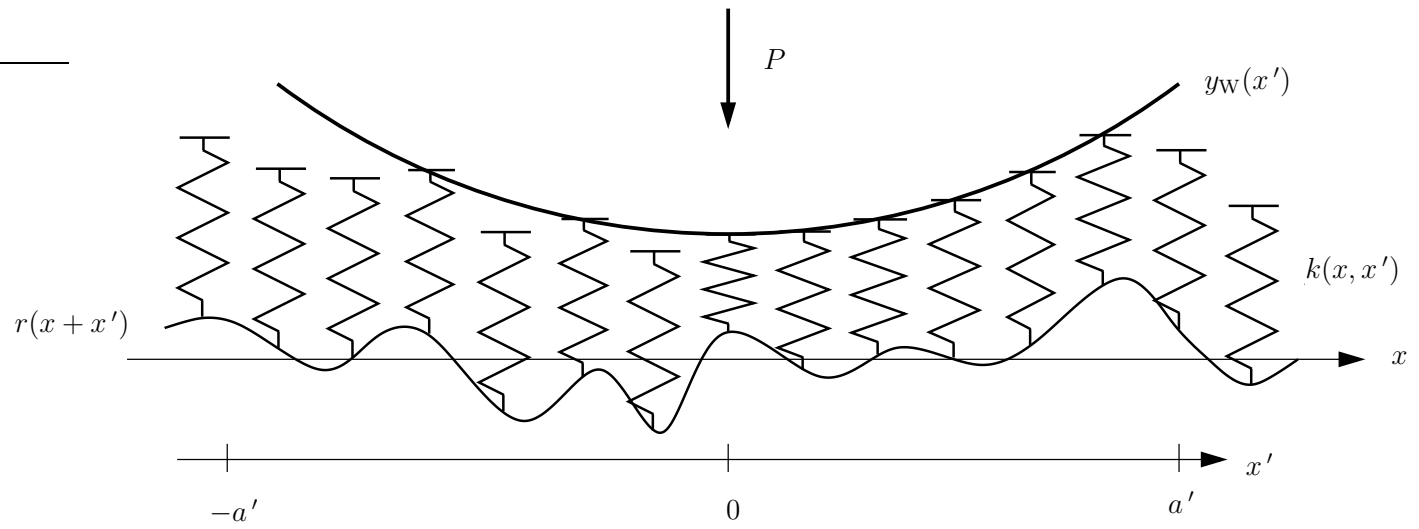

Fig. 3. Bedding model for the wheel/rail contact.

between wheel and rail (see Fig. 3). For simplicity, only the rail is displayed as rough in Fig. 3, but both wheel and rail are considered as rough. The combined roughness is contained in the variable $r(x)$, which is defined positive for an asperity on the rail.

For the wheel positioned at $x$, an additional parallel axis $x^{\prime}$ with origin at $x$ is introduced. The deflection $\Delta \zeta\left(x, x^{\prime}\right)$ of all involved contact springs depends on the wheel deflection $\xi_{\mathrm{W}}(x)$, the rail deflection $\xi_{\mathrm{R}}(x)$, the combined roughness $r\left(x+x^{\prime}\right)$ and the wheel profile $y_{\mathrm{W}}\left(x^{\prime}\right)$

$$
\Delta \zeta\left(x, x^{\prime}\right)=\xi_{\mathrm{W}}(x)-\xi_{\mathrm{R}}(x)+r\left(x+x^{\prime}\right)-y_{\mathrm{W}}\left(x^{\prime}\right) .
$$

The total contact force is obtained by integrating over the bedding

$$
F_{\mathrm{n}}(x)=\int_{-a^{\prime}}^{a^{\prime}} k\left(x, x^{\prime}\right) \Delta \zeta\left(x, x^{\prime}\right) \mathrm{d} x^{\prime}
$$

that has a stiffness per unit length $k\left(x, x^{\prime}\right)$

$$
k\left(x, x^{\prime}\right)=\left\{\begin{array}{ccc}
\frac{1}{2} \frac{E}{\left(1-\nu^{2}\right)} & \text { for } \quad \Delta \zeta\left(x, x^{\prime}\right) \geq 0 \\
0 & \text { for } \quad \Delta \zeta\left(x, x^{\prime}\right)<0
\end{array},\right.
$$

where $E$ is the Young's modulus and $\nu$ the Poisson's ratio of rail and wheel (which are assumed to be of the same material). The integration domain $\left[-a^{\prime}, a^{\prime}\right]$ has to be chosen long enough to include all potential points of contact. Loss of contact can occur for each of the springs in the bedding. This takes place if $\Delta \zeta\left(x, x^{\prime}\right)<0$. In this case, the stiffness $k\left(x, x^{\prime}\right)$ is set to zero.

The choice of $k\left(x, x^{\prime}\right)$ in equation (10) makes it possible that the bedding correctly models Hertz contact for smooth surfaces if, additionally, the wheel radius is adjusted according to

$$
R_{\mathrm{W}}^{\mathrm{m}}=\frac{1}{2} R
$$

where the original radius of curvature $R$ of the conical wheel in rolling direction and the transverse radius of curvature of the straight rail are assumed equal [13]. The profile of the wheel $y_{\mathrm{W}}\left(x^{\prime}\right)$ is approximated by

$$
y_{\mathrm{W}}\left(x^{\prime}\right) \approx \frac{x^{\prime 2}}{2 R_{\mathrm{W}}^{\mathrm{m}}} .
$$


Ford and Thompson [13] applied this type of bedding model for quasi-static contact filtering i.e. determination of an equivalent roughness that accounts for the contact filter effect as a preprocessing step. They called their model two-dimensional 'distributed point reacting springs' (DPRS) model.

Considering the relation

$$
x=v t
$$

the non-linear system of equations (5) to (12) is a complete description of the contact problem. After discretising, the system can be solved for each time step applying e.g. the Newton-Raphson method.

\subsection{Modelling of the non-linear contact stiffness including roughness}

The previously described approach for the modelling of the contact stiffness using a bedding model already has a non-linear character, since the spring stiffness switches to zero as soon as a spring loses contact. An alternative approach is to formulate the total stiffness of the contact $k_{\text {tot }}$ by one single spring with a non-linear characteristics that depends on the roughness of rail and wheel and the moduli of elasticity of both. Mathematically, this reads as

$$
F_{\mathrm{n}}\left(x, \Delta \zeta_{0}\right)=\int_{0}^{\Delta \zeta_{0}} k_{\text {tot }}(x, \sigma) \mathrm{d} \sigma,
$$

where $\Delta \zeta_{0}$ is the compression of the spring. As a thought experiment one could think of the process of pressing a wheel on the rail, which itself is assumed at rest. In the very first moments of contact, only a few asperities of the rough wheel and rail surface will touch each other and form junctions. Forcing the wheel closer to the rail, more and more junctions form and the stiffness increases due to an increasing area of real contact.

There are different ways of obtaining the stiffness $k_{\text {tot }}$ from this process. One procedure could be based on the bedding model presented in section 2.2. The only difference to the previously presented procedure is that the resulting stiffness function for each wheel position $x$ is calculated in advance. Numerically, it is of advantage to work with a resulting contact force as function of the compression of the spring avoiding the integration in equation (14). This force can be calculated by evaluating the integral over the compressed bedding area

$$
F_{\mathrm{n}}\left(x, \Delta \zeta_{0}\right)=\int_{-a}^{a} k\left(x, x^{\prime}\right) \Delta \zeta\left(x, x^{\prime}\right) \mathrm{d} x^{\prime},
$$

where $\Delta \zeta$ is given by the position $\Delta \zeta_{0}$ of the wheel, the combined roughness $r$ of wheel and rail, the stiffness $k$ of the individual springs, and the curvature $y_{\mathrm{W}}$ of the wheel

$$
\Delta \zeta\left(x, x^{\prime}\right)=\Delta \zeta_{0}+r\left(x+x^{\prime}\right)-y_{\mathrm{W}}\left(x^{\prime}\right) .
$$

After calculating these functions $F_{\mathrm{n}}\left(x, \Delta \zeta_{0}\right)$ for each centre point of contact, they can be used in the contact algorithm to determine the force as function of wheel and rail motion. The variable $\Delta \zeta_{0}$ is then the difference between both movements. Using a bedding model for calculating the resulting force, is just one possibility. Alternatives can be found in the literature. Andersson [14] uses e.g. a third body in the form of an elastic layer between tyre and road and calculates the resulting non-linear stiffness as function of the area of real contact. To follow this approach, however, would demand a detailed description of rail and wheel roughness with sufficient resolution. 


\subsection{Inclusion of tangential friction}

The main motivation for the approach discussed in this paper is its potential to include frictional forces in a relatively simple and straightforward manner. This is true for both, longitudinal and lateral friction between wheel and rail. Although the implementation of the model is beyond the scope of this paper, in the following it is briefly shown how to include friction into the model. For simplicity, only one tangential direction t (longitudinal or lateral) is considered.

The starting point is again the description of wheel and rail in the form of Green's functions. To include friction, the set of Green's functions has to be extended. The moving Green's function $\tilde{g}_{\mathrm{R}, v}^{x_{0}}(t)$ describing the response $\xi_{\mathrm{R}, \mathrm{n}}$ of the rail in the normal direction to the rail surface due to a unit force $F_{\mathrm{n}}$ in the normal direction is renamed to $\tilde{g}_{\mathrm{R}, \mathrm{nn}}^{x_{0}}(t)$. Furthermore, a moving Green's function $\tilde{g}_{\mathrm{R}, \mathrm{tt}}^{x_{0}}(t)$ representing the response $\xi_{\mathrm{R}, \mathrm{t}}$ in the tangential direction due to a tangential force $F_{\mathrm{t}}$ is introduced. Normal forces and tangential forces will also lead to tangential and normal displacements, respectively, which is expressed by the moving Green's functions $\tilde{g}_{\mathrm{R}, \mathrm{nt}}^{x_{0}}(t)$ and $\tilde{g}_{\mathrm{R}, \mathrm{tn}}^{x_{0}}(t)$. In most of the cases, this coupling between normal and tangential directions cannot be neglected. A similar set of Green's functions are needed for the response of the wheel. For both wheel and rail, one can establish a set of equations as

$$
\begin{aligned}
& \xi_{\mathrm{R}, \mathrm{n}}(t)=F_{\mathrm{n}}(t) * \tilde{g}_{\mathrm{R}, \mathrm{nn}}^{x_{0}}(t)+F_{\mathrm{t}}(t) * \tilde{g}_{\mathrm{R}, \mathrm{tn}}^{x_{0}}(t) \\
& \dot{\xi}_{\mathrm{R}, \mathrm{t}}(t)=F_{\mathrm{n}}(t) * \dot{\tilde{g}}_{\mathrm{R}, \mathrm{nt}}^{x_{0}}(t)+F_{\mathrm{t}}(t) * \dot{\tilde{g}}_{\mathrm{R}, \mathrm{tt}}^{x_{0}}(t) \\
& \xi_{\mathrm{W}, \mathrm{n}}(t)=-F_{\mathrm{n}}(t) * \tilde{g}_{\mathrm{W}, \mathrm{nn}}(t)-F_{\mathrm{t}}(t) * \tilde{g}_{\mathrm{W}, \mathrm{tn}}(t)+\xi_{\mathrm{S}, \mathrm{n}} \\
& \dot{\xi}_{\mathrm{W}, \mathrm{t}}(t)=-F_{\mathrm{n}}(t) * \dot{\tilde{g}}_{\mathrm{W}, \mathrm{nt}}(t)-F_{\mathrm{t}}(t) * \dot{\tilde{g}}_{\mathrm{W}, \mathrm{tt}}(t),
\end{aligned}
$$

where the dot denotes a time derivative.

The only missing part is the relation between normal displacements of wheel and rail and the normal force as well as the relation between the relative tangential velocity between wheel and rail and the tangential force. The first relation can e.g. be taken from section 2.3 while for the latter the friction characteristics as function of relative velocity

$$
F_{\mathrm{t}}(t)=F_{\mathrm{n}}\left(x(t), \Delta \zeta_{0}\right) \mu(\Delta u(t))
$$

can be applied. The relative velocity is here defined as $\Delta u(t)=\dot{\xi}_{\text {,Wt }}(t)-\dot{\xi}_{\mathrm{R}, \mathrm{t}}(t)$.

As a result, a non-linear equation system is obtained which besides its higher complexity is similar to that for normal contact forces only. In the same manner, it can be solved for each time step by a Newton-Raphson procedure. Although the model is expected to work fine and numerically efficient, it has to be stated that the critical point certainly is obtaining adequate input data, such as the friction characteristics $\mu(\Delta u(t))$ for the wheel/rail contact.

\section{Validation of the model against an existing time domain model}

For validation, simulation results of the normal interaction model described in section 2.2 are compared to results from the train/track interaction model DIFF [10]. In this model, developed by Nielsen and Igeland, the transient vertical interaction problem is solved by use of an extended statespace vector approach in conjunction with a complex modal superposition for the track. DIFF itself has been validated by field testing [11], where good agreement between calculated and measured vertical contact forces was observed. 
Some simplifications are introduced in the proposed model and in DIFF in order to facilitate the comparison:

- The wheel model is replaced by an unsprung mass $M$ without suspension. Equations (5) and (6) hereby reduce to

$$
M \ddot{\xi}_{\mathrm{W}}(t)=P-F_{\mathrm{n}}(t) .
$$

- The Winkler bedding (Fig. 3) is substituted by one single contact spring modelling Hertz contact. Equations (9) and (10) are consequently simplified to

$$
F_{\mathrm{n}}(x)=\left\{\begin{array}{cl}
C_{\mathrm{H}}(\Delta \zeta(x))^{\frac{3}{2}} & \text { for } \quad \Delta \zeta(x) \geq 0 \\
0 & \text { for } \Delta \zeta(x)<0
\end{array}\right.
$$

where

$$
C_{\mathrm{H}}=\frac{2}{3} \frac{E}{1-\nu^{2}} \sqrt{R}
$$

is the Hertzian constant. Furthermore, wheel and railhead radius of curvature $R$ are taken as equal, which implies that the contact patch is assumed circular.

- In order to include the contact filter effect, a quasi-static filtering of the roughness $r(x)$ is performed in a preprocessing step. An equivalent roughness $r_{\text {eq }}(x)$ is calculated for each position $x$ by averaging the roughness over the nominal contact patch length. The nominal contact patch length is obtained from the Hertzian law for $F_{\mathrm{n}}=P$.

The parameters applied in the simulations are listed in Tab. 1. Parameters of the track model are given in reference [11].

Tab. 1. Simulation parameters.

\begin{tabular}{cll}
\hline \hline Parameter & Value & Description \\
\hline$E$ & $210 \mathrm{GPa}$ & Young's modulus of wheel and rail \\
$\nu$ & 0.283 & Poisson's ratio of wheel and rail \\
$R$ & $0.46 \mathrm{~m}$ & Wheel and railhead radius of curvature \\
$P$ & $65 \mathrm{kN}$ & Static preload \\
$v$ & $100 \mathrm{~km} / \mathrm{h}$ & train speed \\
\hline \hline
\end{tabular}

For the calculation of the moving Green's functions representing the track, receptances in the frequency range 0 to $4 \mathrm{kHz}$ have been used. In order to include the same frequency content in DIFF, the lowest 261 modal pairs are accounted for in the modal synthesis performed for the track.

Comparisons are carried out for different roughness excitations and the results are displayed in Fig. 4 in the form of discrete Fourier transforms of the calculated normal contact force. The cases considered are a perfect rail and wheel, a rail with sinusoidal corrugation with wavelength $\lambda=0.015 \mathrm{~m}$ and amplitude $A=10 \mu \mathrm{m}$ and a roughness profile calculated from measured data. The measured roughness data was available in terms of a third-octave band spectrum measured on a corrugated rail at the test site Vretstorp on the line Göteborg-Stockholm and a mean of five third-octave band spectra measured on X2 trailer wheels. Details about the measurements can be found in references [11] and [15]. From the combined wheel/rail roughness spectrum, a sample of an energy-equivalent roughness sequence was generated by a sum of sine functions with contributions from the third-octave 


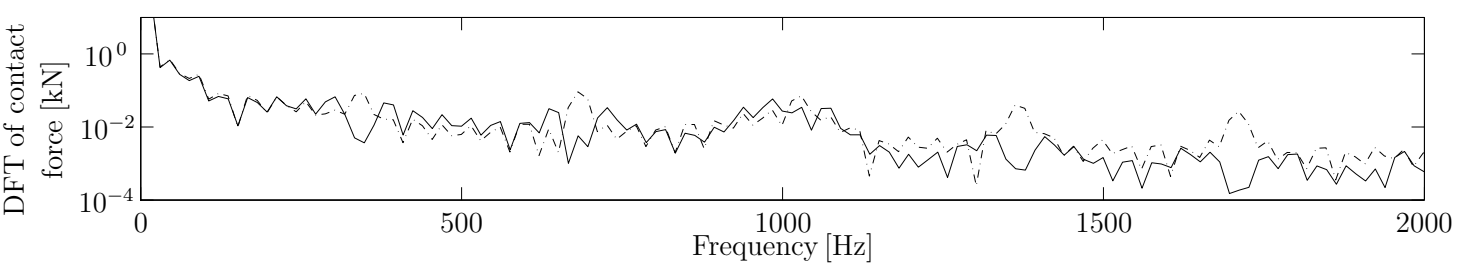

(a) Perfect rail and wheel.

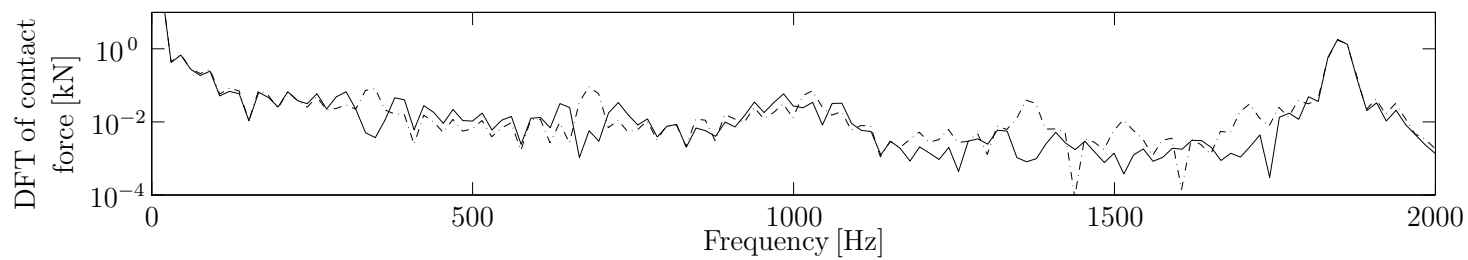

(b) Rail with sinusoidal corrugation $\lambda=0.015 \mathrm{~m}$ and $A=10 \mu \mathrm{m}$, perfect wheel.

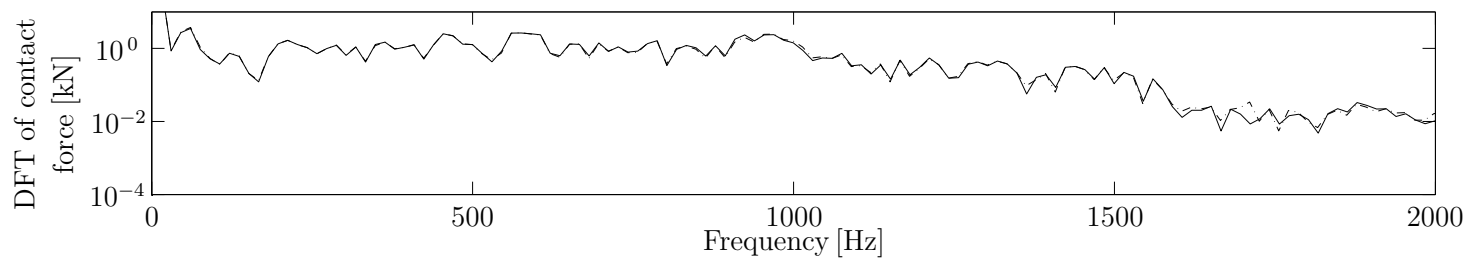

(c) Corrugated rail and moderately rough wheel. Data from measured third-octave band spectra.

Fig. 4. Discrete Fourier transform of the contact force for different roughness excitations. Comparison between the proposed model ( $\longrightarrow$ ) and DIFF $(-\cdot-\cdot)$. Train speed $v=100 \mathrm{~km} / \mathrm{h}$ and wheel and rail radius of curvature $R=0.46 \mathrm{~m}$.

bands with centre wavelengths in the interval $2-250 \mathrm{~cm}$. The amplitudes of the sine functions were determined by the roughness level in each third-octave band, while phase angles were taken from a uniform random distribution between 0 and $2 \pi$ [16]. It should be noted that the roughness sequence generated by this means differs from the original roughness sequence at the test site and does not necessarily generate the same contact forces as the original roughness.

For all cases, the agreement between the proposed model and DIFF is very good. In Fig. 4(a) and 4(b) additional, periodically appearing, peaks can be observed for the DIFF simulations. These correspond to the element passing frequency (and multiples) in the finite-element model of the track. While deflection is continuous over the element boundaries, the slope of the deflection is noncontinuous and provides additional parametric excitation to the system.

The simulations show that the proposed model is also very time efficient. Provided that the Green's functions for the rail are precalculated, the computation time for one simulation is typically less than 20 seconds on a PC with a Pentium $2.0 \mathrm{GHz}$ processor.

\section{Evaluation of the contact filter effect}

The normal interaction model described in section 2.2 in combination with the wheel model of section 3 (unsprung mass) is now applied to perform simulations of the wheel passing over a rail with sinusoidal corrugation. The corrugation is assumed to consist of only one sine curve with corrugation wavelength $\lambda$ and amplitude $A$. Since the bedding model incorporates several springs over the contact 


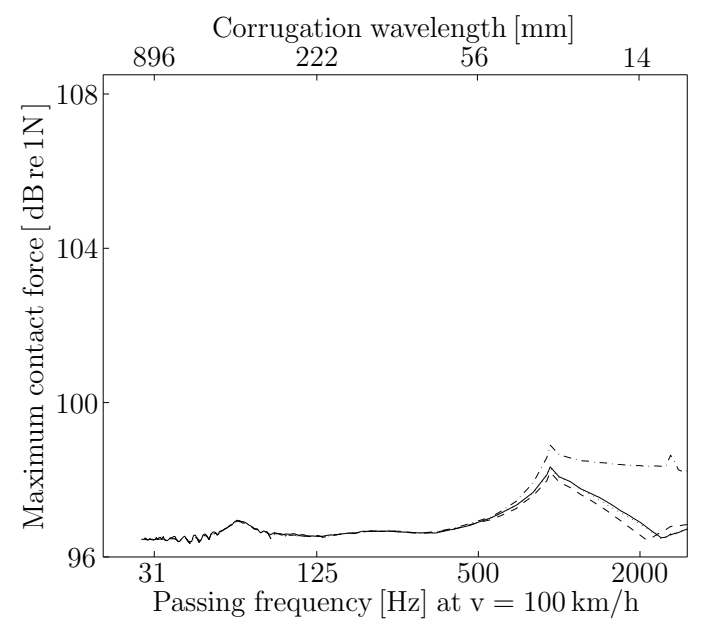

(a) Corrugation amplitude $\mathrm{A}=10 \mu \mathrm{m}$.

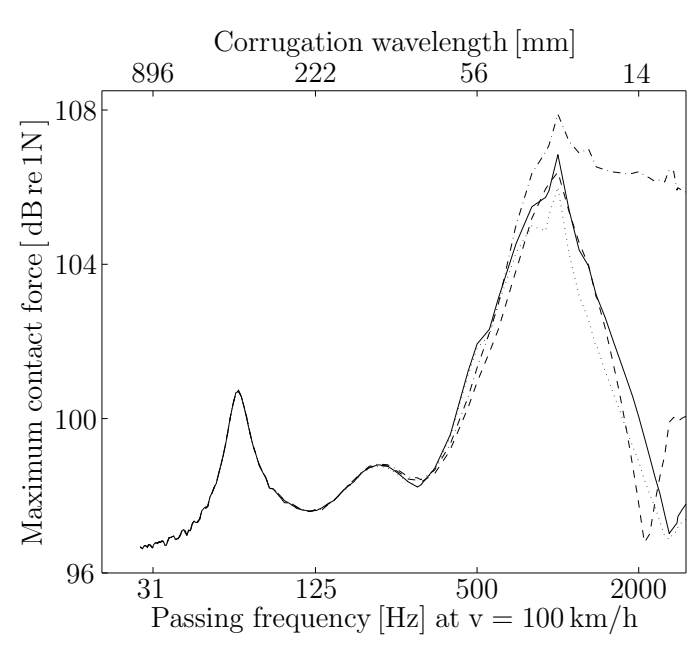

(b) Corrugation amplitude $\mathrm{A}=100 \mu \mathrm{m}$.

Fig. 5. Maximum contact force for passing over a rail with sinusoidal corrugation. — quasi-static roughness filtering with Winkler bedding, --- quasi-static roughness filtering by averaging, $-\cdot-\cdot$ no roughness filtering, $\cdots \cdots$ dynamic roughness filtering.

patch length, high-frequency excitation that has wavelengths in the order of magnitude of the contact patch length or shorter is attenuated. The model also accounts for a time-variant contact patch length during the simulations and can therefore be said to perform a dynamic roughness filtering. For comparison, the same simulations are carried out with the simpler one-spring model of section 3 , which does not include dynamic roughness filtering. In order to account for the contact filtering effect in this model, an equivalent roughness is calculated as a preprocessing step, which is called quasi-static roughness filtering. Three such methods are considered:

- An equivalent roughness is calculated by means of the Winkler bedding depicted in Fig. 3. The procedure is described in detail in reference [13].

- Averaging over the contact patch length like in section 3 is applied to obtain an equivalent roughness.

- No equivalent roughness is calculated and the simulations are carried out with the original roughness excitation.

The results of the simulations with the four different interaction models are shown in Fig. 5 in the form of the maximum contact force under steady state conditions as function of the corrugation wavelength. Steady state conditions are assumed to be reached when the wheel has passed the first ten sleeper spans. The corrugation amplitudes $10 \mu \mathrm{m}$ and $100 \mu \mathrm{m}$ are considered in Figs. 5(a) and 5(b), respectively.

Three distinct peaks are observed in Fig. 5(b) and to a less clear extent also in Fig. 5(a). These peaks roughly correspond to the peaks in the track receptance (see Fig. 1(a)), but due to the coupling between wheel and rail the exact location of the resonance frequencies pertaining to the compound system differs from those of the rail.

The contact filter effect is clearly noticeable for corrugation wavelengths that are up to three to four times the nominal contact patch length (the latter being $10.7 \mathrm{~mm}$ for the parameters listed in Tab. 1). In both figures, the curve corresponding to no roughness filtering systematically gives rise to 
higher contact forces in this wavelength range. However, not much difference is observed between the different methods of roughness filtering. The simple averaging filtering performs surprisingly well. In Fig. 5(a) the difference between averaging filtering and dynamic filtering is negligible and in Fig. 5(b) it reaches slightly over $3 \mathrm{~dB}$ for high frequencies. No significant difference is observed between quasi-static filtering with the Winkler bedding and dynamic filtering. The results could look substantially different for frequencies above $3000 \mathrm{~Hz}$, but the presented simulations are limited by the frequency range of validity of the applied track model.

The results could also look different if a measured roughness sequence is applied as excitation.

\section{Conclusions}

A numerical time-stepping model to predict the wheel/track dynamic interaction has been presented. The wheel is represented by Green's functions and the track by moving Green's functions. The model includes a non-linear contact spring, allows for loss of contact and accounts for parametric excitation due to space-dependent track stiffness.

The normal interaction model has been validated by comparison with results from the dynamic train/track interaction model DIFF [10]. The excitation cases, perfect track, rail with sinusoidal corrugation and roughness sequence calculated from measured third-octave band roughness spectra, were considered. Very good agreement was obtained for all the investigated cases.

The model has also been applied to evaluate the contact filter effect for passing over a rail with sinusoidal corrugation and to compare different roughness filtering methods. For the considered cases, no significant difference was observed between dynamic and quasi-static roughness filtering based on a Winkler bedding in the frequency range up to $3000 \mathrm{~Hz}$. Even the simple averaging filtering performed sufficiently well. However, the results could look substantially different for higher frequencies and/or when applying a measured roughness profile as excitation input.

One major advantage of the presented interaction model is its high computational efficiency. Even if combined with a complex FE-model of the track, the calculation time for a simulation is typically less than 20 seconds on a PC with a Pentium $2.0 \mathrm{GHz}$ processor. This can be attributed to the fact that the Green's functions are precalculated and, by this means, wheel, rail and contact calculations are separated. One drawback is that new moving Green's functions for the rail have to be calculated for each train speed.

When implementing the approach presented for the non-linear contact stiffness based on the area of real contact, detailed roughness information can be processed because the stiffness functions can be precalculated for each wheel position. To follow this approach, however, would demand that a detailed description of rail and wheel roughness with sufficient resolution is available.

Another important advantage of the presented interaction model, being at the same time the main motivation for its development, is its potential to include frictional forces in a relatively simple and straightforward manner. This is true for both, longitudinal and lateral friction between wheel and rail.

\section{Acknowledgements}

The work described has been performed within the project "Generation of External Noise from Trains" (VB10) and forms part of the activities in the Centre of Excellence CHARMEC (CHAlmers Railway MEChanics, www.charmec.chalmers.se). It is funded by CHARMEC in cooperation with VINNOVA, the Swedish Governmental Agency for Innovation Systems. 


\section{References}

[1] P.J. Remington. Wheel/rail rolling noise, I: Theoretical analysis. Journal of the Acoustical Society of America, 81(6):1824-1832, 1987.

[2] D.J. Thompson. Wheel-rail noise generation, part I: Introduction and interaction model. Journal of Sound and Vibration, 161(3):387-400, 1993.

[3] T.X. Wu and D.J. Thompson. Theoretical investigation of wheel/rail non-linear interaction due to roughness excitation. Vehicle System Dynamics, 34:261-282, 2000.

[4] T.X. Wu and D.J. Thompson. A hybrid model for the noise generation due to railway wheel flats. Journal of Sound and Vibration, 251(1):115-139, 2002.

[5] T.X. Wu and D.J. Thompson. On the impact noise generation due to a wheel passing over rail joints. Journal of Sound and Vibration, 267:485-496, 2003.

[6] A. Nordborg. Journal of the Acoustical Society of America.

[7] T.X. Wu and D.J. Thompson. Wheel/rail non-linear interactions with coupling between vertical and lateral directions. Vehicle System Dynamics, 41(1):27-49, 2004.

[8] F. Wullens and W. Kropp. A three dimensional contact model for tyre/road interaction in rolling conditions. Acta Acustica united with Acustica, 90(4):702-711, 2004.

[9] M. Heckl. Proposal for a railway simulation program. In A Workshop on Rolling Noise Generation, pages 128-148. Institut für Technische Akustik, Technische Universität Berlin, 1989.

[10] J.C.O Nielsen and A. Igeland. Vertical dynamic interaction between train and track - influence of wheel and track imperfections. Journal of Sound and Vibration, 187(5):825-839, 1995.

[11] J.C.O Nielsen. High-frequency vertical wheel-rail contact forces - validation of a prediction model by field testing. In Proceedings of the Seventh International Conference on Contact Mechanics and Wear of Rail/Wheel Systems, Brisbane, Australia, September 2006.

[12] J.C.O. Nielsen. Dynamic interaction between wheel and track - a parametric search towards an optimal design of rail structures. Vehicle System Dynamics, 23(2):115 - 132, 1994.

[13] R.A.J. Ford and D.J. Thompson. Simplified contact filters in wheel/rail noise prediction. Journal of Sound and Vibration, 293:807-818, 2006.

[14] P.B.U. Andersson. Modelling non-linear contact stiffness in tyre/road contact. In Proceedings of the 19th International Conference on Acoustics, Madrid, Spain, September 2007.

[15] A. Johansson. Out-of-round railway wheels - assessment of wheel tread irregularities in train traffic. Journal of Sound and Vibration, 293(3-5):795-806, 2006.

[16] M. Hiensch, J.C.O Nielsen, and E. Verheijen. Rail corrugation in The Netherlands - measurements and simulations. Wear, 253:140-149, 2002. 